\title{
Tratamento cirúrgico da limitação de abertura bucal causada por hiperplasia do processo coronoide: relato de caso
}

\section{Surgical treatment for mouth opening limitation caused by coronoid process hyperplasia: case report}

\author{
Caroline Klabunde* \\ João Batista Burzlaff* \\ Vinicius Salim Silveira** \\ Leonardo Tonietto ${ }^{* * *}$ \\ Jéssica Lemos Gulinelli***** \\ Thiago Calcagnotto ${ }^{* * * * *}$
}

\section{Resumo}

A hiperplasia do processo coronoide é uma condição incomum de etiologia desconhecida que se apresenta clinicamente por meio da limitação de abertura bucal e não possui sintomatologia dolorosa durante a abertura e o fechamento bucal. Objetivo: relatar e discutir, por meio de um caso cirúrgico, o tratamento da limitação de abertura bucal causada por hiperplasia bilateral do processo coronoide. Relato de caso: paciente do sexo feminino, com 11 anos de idade, foi encaminhada para atendimento devido à dificuldade de mastigação em função da limitação de abertura bucal, sem histórico de trauma em face ou na região articular. O exame tomográfico evidenciou o alongamento bilateral do processo coronoide, fazendo com que ele colidisse com o arco zigomático durante a abertura bucal e causasse o travamento. O tratamento proposto foi a coronoidectomia bilateral com acesso cirúrgico intraoral, obtendo no pós-cirúrgico imediato um ganho na abertura bucal. Considerações finais: a coronoidectomia é uma abordagem cirúrgica de fácil acesso por via intraoral, pouco traumática e eficaz no tratamento de pacientes com hiperplasia do processo coronoide.

Palavras-chave: Coronoidectomia. Hiperplasia do processo coronoide. Limitação de abertura bucal.

\section{Introdução}

A hiperplasia do processo coronoide da mandíbula é uma condição incomum, definida como um alongamento anormal do processo coronoide, mas formado por tecido ósseo histologicamente normal. A principal característica clínica é a limitação da abertura bucal, que pode ocorrer de forma lenta e progressiva, embora nem todos os pacientes apresentem como queixa principal ${ }^{1,2}$. Não apenas a abertura bucal, mas também os movimentos mandibulares de protrusão e lateralidade se tornam limitados como resultado do contato do processo coronoide com a superfície do arco zigomático. Geralmente, não há história de dor ou trauma associada a esse crescimento atípico ${ }^{3}$.

A hiperplasia do processo coronoide pode ser uni ou bilateral, sendo ambas as condições mais prevalentes em homens. Casos de hiperplasia unilateral podem estar associados à ocorrência de assimetria facial $^{1,4}$.

A etiologia, embora já postuladas várias teorias, como disfunção da articulação-temporomandibu$l^{2}{ }^{2}$, anquilose, traumas, estímulos endócrinos ${ }^{4}$, hiDental CEEO.

*** Doutor em CTBMF, Pontifícia Universidade Católica do Rio Grande do Sul (PUCRS). Professor do Curso de Especialização em CTBMF, Fatec Dental CEEO. Doutor em CTBMF, PUCRS. Professor do Curso de Especialização em CTBMF, Fatec Dental CEEO.

***** Doutora em CTBMF, Universidade Estadual Paulista. Professor do Curso de Pós-Graduação em CTBMF, Universidade Sagrado Coração.

** Mestre em CTBMF, Ufrgs. Coordenador do Curso de Especialização em CTBMF, Fatec Dental CEEO. 
peratividade muscular do músculo temporal ${ }^{5}$ e até mesmo fatores genéticos ${ }^{6}$, ainda permanece desconhecida ${ }^{1}$.

O diagnóstico pode ser difícil devido ao foco clínico nas articulações temporomandibulares ${ }^{3}$, sendo que a incapacidade de abrir completamente a boca tende a desviar a atenção para a articulação ${ }^{4}$. Segundo autores ${ }^{2}$ que avaliaram pacientes com limitação de abertura bucal sem etiologia definida, há uma frequência de $5 \%$ de hiperplasia do processo coronoide como agente causal, indicando um subdiagnóstico da condição.

Há, na literatura, relatos de ressecções realizadas via extrabucal e também via submandibular como método de intervenção ${ }^{1,7}$. No entanto, a coronoidectomia por via intrabucal tem sido considerada o tratamento de eleição para os casos de hiperplasia do processo coronoide ${ }^{3,5}$. A fibrose induzida cirurgicamente e a tendência a um novo crescimento do processo coronoide tornam as expectativas em relação ao tratamento frustrantes e, por isso, muitas vezes, o tratamento fisioterápico é associado no pós-operatório imediato ${ }^{1}$.

Assim, o presente trabalho tem como objetivo relatar a abordagem cirúrgica para o tratamento de um caso de limitação de abertura bucal causada por hiperplasia bilateral do processo coronoide, ilustrando a manutenção dos resultados após três anos.

\section{Relato do caso}

Uma paciente do sexo feminino, com 11 anos de idade, foi encaminhada para avaliação com cirurgião bucomaxilofacial devido à dificuldade de mastigação e alimentação em função de limitação de abertura bucal. Na primeira consulta, sem exames de imagem, o exame clínico evidenciou limitação de abertura bucal de apenas $17 \mathrm{~mm}$ (Figura 1), mesmo com uso de estimulação por meio de força manual externa. Observaram-se, ainda, movimentos de abertura e fechamento bucal sem desvios de linha. A anamnese da paciente, conforme relatado pelo responsável, não apresentava histórico de traumas em face ou na região articular que pudesse representar significância em termos de recordação. O parto foi normal, mas com período gestacional de 7 meses, e a gestante, durante esse período, era dependente química, fazendo uso de cocaína, maconha, cigarro e álcool. A paciente não apresentava sinais clínicos de déficit cognitivo ou alterações morfológicas.

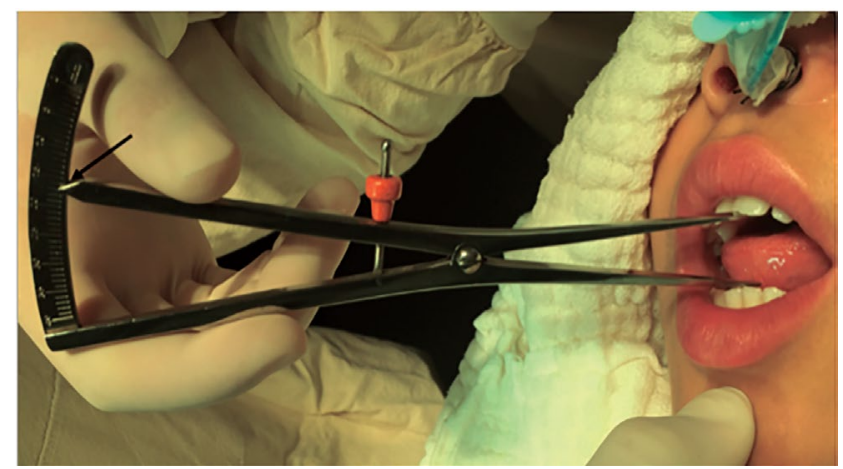

Figura 1 - Imagem clínica pré-operatória evidenciando, com auxílio de compasso de ponta seca, abertura bucal de $17 \mathrm{~mm}$ (seta indicando a medida de $17 \mathrm{~mm}$ )

Fonte: autores

$\mathrm{O}$ exame clínico associado somente à anamnese, devido à inexistência de exames de imagem na primeira consulta, levou à suspeita clínica da existência de alguma alteração de ordem articular (alteração morfológica das cabeças da mandíbula, hiperplasia condilar, anquilose, deslocamento de disco articular com travamento) que pudesse causar a limitação do processo de abertura e fechamento bucal e, para confirmação, foi solicitada, no primeiro momento, uma tomografia bilateral das regiões das articulações temporomandibulares.

A imagem tomográfica (Figura 2), avaliada na segunda consulta, evidenciou espaço articular dentro dos padrões de normalidade, cabeças da mandíbula sem alterações (sem áreas de absorção, hipertrofia ou anquilose).

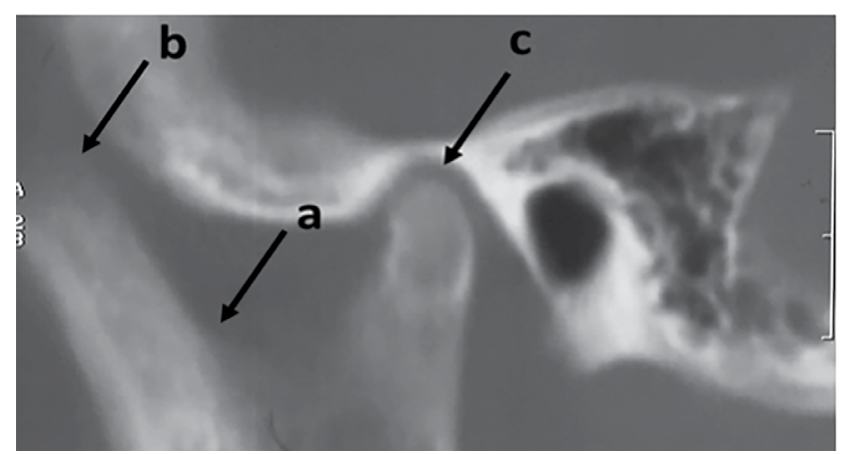

Figura 2 - Imagem tomográfica pré-operatória em corte sagital da região de ATM evidenciando hiperplasia do processo coro-

Legenda: a) base do processo coronoide; b) porção final do processo coronoide, em que há inserção das fibras ligamentares do músculo temporal; c) cabeça da mandíbula sem alterações morfológicas e com espaço articular mantido.

Fonte: autores. 
O processo coronoide, no entanto, apresentava-se hiperplásico (alongado) e, durante a abertura bucal, seu movimento em direção à fossa temporal fazia com que este colidisse com o arco zigomático, limitando a abertura bucal. $\mathrm{O}$ diagnóstico estabelecido para o quadro apresentado pela paciente foi de limitação de abertura bucal causada por hiperplasia do processo coronoide, tendo como proposta de tratamento a realização cirúrgica de coronoidectomia bilateral.

O procedimento cirúrgico foi realizado sob anestesia geral por meio de intubação nasotraqueal. Após a assepsia facial e intrabucal, realizou-se infiltração local na região de ramo ascendente e na região de processo coronoide, com $5 \mathrm{ml}$ de solução de cloridrato de bupivacaína 5\% com epinefrina
1:200.000 (Neocaína 0,5\%, Cristália Produtos Químicos Farmacêuticos LTDA., São Paulo, Brasil) em cada lado da paciente. Ainda, na região ascendente de ramo, sobre a linha oblíqua, foi realizada uma incisão de cerca de $3,5 \mathrm{~cm}$ com bisturi elétrico (Figura 3A). O descolamento, inicialmente, foi realizado com descoladores de Molt 1/4 para a porção superior em direção ao processo coronoide até a localização da incisura mandibular, por meio de descoladores curvos de Molt. Após a localização da incisura, as inserções ligamentares do músculo temporal no processo coronoide foram desinseridas, e o processo coronoide foi então removido cirurgicamente desde a sua base por meio de osteotomias com uso de sistema Piezzo de corte (Figura 3B). Os processos coronoides, de ambos os lados, foram então removidos (Figura 3C).

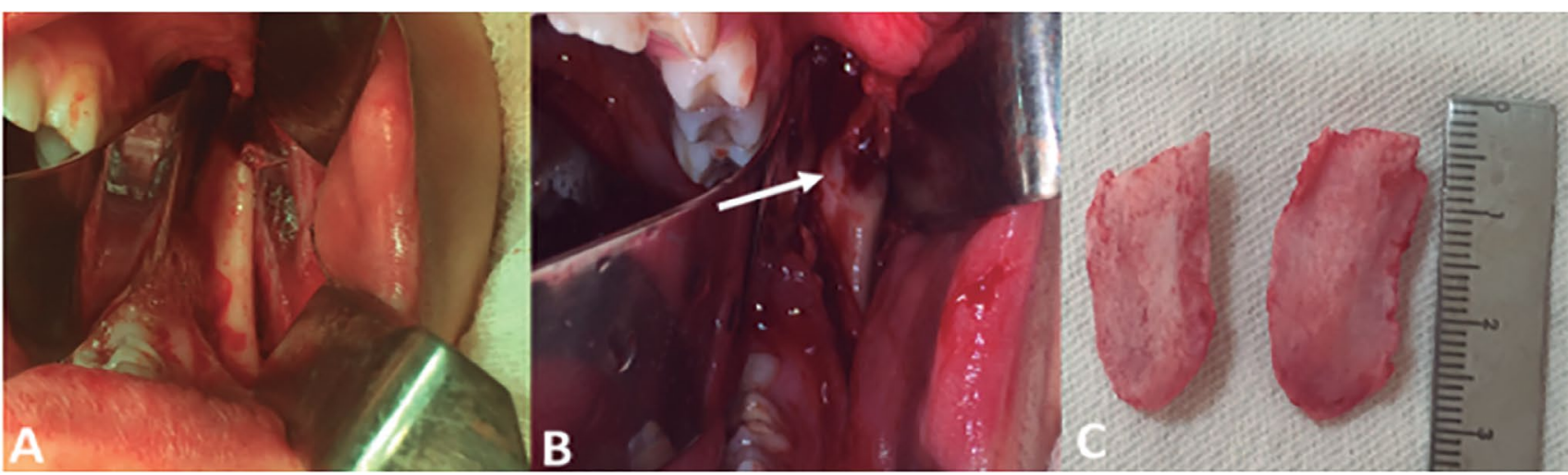

Figura 3 - Imagem clínica transoperatória evidenciando procedimento cirúrgico de coronoidectomia bilateral

Legenda: a) incisão na região de ramo ascendente com exposição da base do processo coronoide; b) seta indicando o local de osteotomia com sistema Piezzo de corte; c) processos coronoides removidos.

Fonte: autores.

Após a remoção dos processos coronoides hiperplásicos, ainda com a paciente sob anestesia geral, realizou-se a abertura bucal sem excesso de força, observando-se $35 \mathrm{~mm}$ de abertura bucal, um ganho de $18 \mathrm{~mm}$ em relação ao quadro clínico inicial.

Os cuidados com a ferida cirúrgica envolveram lavagem abundante da ferida cirúrgica com solução fisiológica e sutura contínua com fio absorvível à base de poliglactina (Vicryl® Ethicon, Jhonson \& Jhonson do Brasil, São Paulo, Brasil). Os cuidados pós-operatórios envolveram o uso de antibióticos, analgésicos e anti-inflamatórios, em dois momentos: o primeiro momento, durante a internação hospitalar por 24 horas, envolveu administração medicamentosa por via endovenosa de Ampicilina $1.000 \mathrm{mg}$ a cada 8 horas, Dipirona $1.000 \mathrm{mg}$ a cada 8 horas e Dexametasona na dose de $8 \mathrm{mg}$ a cada 12 horas. No segundo momento, após alta melhorada para casa, o controle medicamentoso pós-operatório foi realizado por meio do uso de Amoxicilina $875 \mathrm{mg}$ associada à Clavulanato de potássio $125 \mathrm{mg}$, por via oral, a cada 12 horas por 7 dias. A analgesia envolveu o uso de Paracetamol $750 \mathrm{mg}$, por via oral, a cada 6 horas por 3 dias, e o uso de anti-inflamatório com Dexametasona, na dose de $4 \mathrm{mg}$ por via oral a cada 12 horas por 5 dias.

O controle pós-operatório de 7 e 15 dias evidenciou limitação de abertura bucal devido ao edema causado pela cirurgia. Após 30 dias da realização da cirurgia, a abertura bucal era de $35 \mathrm{~mm}$. O controle clínico e tomográfico pós-operatório de três anos evidenciou a presença de $36 \mathrm{~mm}$ de abertura bucal (Figura 4), caracterizando adequados diagnóstico e tratamento, com o sucesso da técnica cirúrgica empregada.

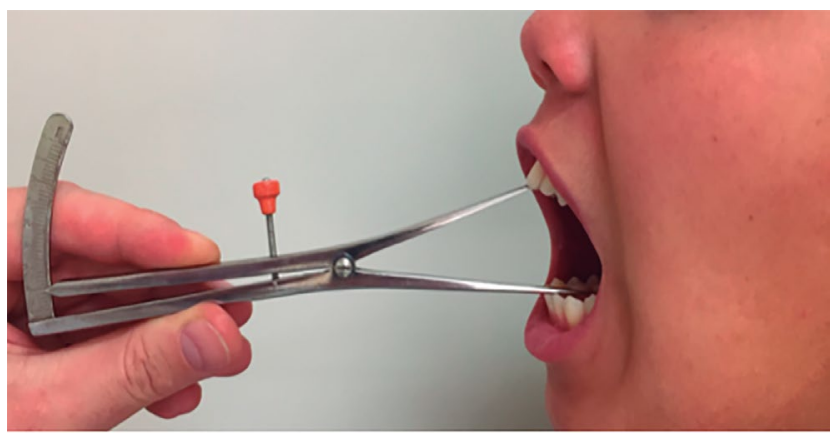

Figura 4 - Imagem clínica pós-operatória de três anos evidenciando abertura bucal da paciente mantida em $36 \mathrm{~mm}$

Fonte: autores 


\section{Discussão}

A hiperplasia bilateral do processo coronoide apresenta prevalência no sexo masculino, sendo relatada na proporção 5:1 entre homens e mulheres, e ocorre principalmente no período da puberdade, que é quando o processo coronoide atinge um crescimento capaz de limitar a abertura bucal ${ }^{1}$.

$\mathrm{O}$ caso relatado neste artigo acomete uma paciente do sexo feminino justamente no período inicial da puberdade, fase de maior incidência ${ }^{8}$. O aumento bilateral do processo coronoide caracteriza a limitação da movimentação mandibular em todas as direções, sem nenhum outro sinal de anormalidade $^{3}$, da mesma forma como o encontrado no caso relatado, que foi tratado de forma cirúrgica por acesso intraoral, preconizando uma abordagem cirúrgica mais conservadora e com menor risco de danos a estruturas nobres, como vasos e nervos.

A limitação progressiva na abertura bucal é o principal sinal de hiperplasia do processo coronoi$\mathrm{de}^{1,3}$. No entanto, ela pode ocorrer em outras situações, como traumatismos faciais, trismo ${ }^{4}$, anquilose $\operatorname{articular}^{5}$, fraturas mandibulares, processos infec$\operatorname{ciosos}^{9} \mathrm{e}$ doenças nas articulações temporomandibulares ${ }^{10}$.

No caso apresentado, devido à ausência de exames complementares na primeira consulta, houve a suspeita clínica de alterações de ordem articular, visto que não havia histórico de trauma relatado pelo responsável, fato que demonstra a importância de exames de imagem como métodos complementares de diagnóstico. Esses exames, por sua vez, descartaram alterações de ordem articular e evidenciaram o alongamento do processo coronoide bilateralmente, diagnosticando a limitação de abertura bucal como decorrente de uma hiperplasia dos processos coronoides.

Sendo assim, os exames radiográficos são importantes tanto para o diagnóstico como para o seguimento do tratamento cirúrgico desta condição, que pode ser facilmente ignorada por não ser frequentemente vista na prática clínica. No entanto, as radiografias panorâmicas não são os melhores exames para detectar realmente o tamanho e a forma dos processos coronoides, uma vez que a sobreposição de imagens nessa região pode levar à dificuldade de visualização adequada das estruturas ósseas. Nesse sentido, a tomografia computadorizada revela mais precisamente a localização e a morfologia das estruturas articulares e adjacentes à articulação ${ }^{11}$.

Após estabelecer o diagnóstico, com base nas características clínicas e imaginológicas, o tratamento proposto foi a realização de coronoidectomia bilateral por meio de acesso cirúrgico intraoral, visando à remoção dos fatores limitantes (processos coronoides alongados), permitindo que a abertura bucal da paciente alcançasse valores fisiológicos e devolvendo as capacidades mastigatória e fonética adequadas.

O tratamento planejado para o caso relatado, por meio de coronoidectomia, é unânime nos casos encontrados na literatura ${ }^{3,5,9}$. No caso descrito, o acesso cirúrgico foi realizado por via intraoral, sendo o tratamento mais comumente empregado, embora existam trabalhos que façam a abordagem cirúrgica por via extraoral, com as justificativas de maior facilidade em se alcançar o músculo temporal, melhor visibilidade e possibilidade de ressecção das fibras que se inserem no processo coronoide ${ }^{1}$. Contudo, a opção pelo acesso intraoral permite maior capacidade de preservação de estruturas nobres, como vasos e nervos, ausência de cicatriz em face e menor morbidade pós-operatória, ainda mais no caso de uma paciente muito jovem.

Além do tratamento cirúrgico, a literatura enfatiza a necessidade de fisioterapia associada no pós-operatório imediato, a fim de ampliar o sucesso do tratamento realizado ${ }^{1}$. Os exercícios necessários de alongamento da musculatura podem ser doloridos, fazendo que com o paciente não os realize de maneira correta, o que pode comprometer o resultado final do tratamento. Assim, é essencial que o paciente e seus familiares sejam esclarecidos sobre a importância das duas etapas para a obtenção do melhor desfecho do caso.

No caso apresentado, na consulta inicial, a paciente apresentava apenas $17 \mathrm{~mm}$ de abertura bucal, situação imediatamente alterada após o tratamento cirúrgico (atingindo uma abertura de $35 \mathrm{~mm}$, representando um ganho de $18 \mathrm{~mm}$ ), o que se manteve ao longo do período pós-operatório de três anos.

A recidiva da hiperplasia do processo coronoide tem sido um achado relativamente comum ${ }^{1}$, portanto, o acompanhamento do paciente é fundamental, com o propósito de observar se há aumento ou redução da limitação bucal com o passar do tempo, sinalizando a retomada do crescimento do processo coronoide, a calcificação de fibras musculares ou até mesmo algum processo patológico articular que possa ter se desenvolvido de forma paralela.

O caso apresentado e a sua resolução por meio de cirurgia, após três anos de acompanhamento, evidenciaram a manutenção do ganho de abertura bucal, uma vez que não houve redução e a paciente manteve uma abertura bucal de $36 \mathrm{~mm}$, ou seja, um milímetro a mais do que o obtido no pós-cirúrgico imediato.

A limitação de abertura bucal devido à hiperplasia do processo coronoide pode ser confundida com outras condições clínicas, levando o paciente a buscar diversos profissionais, o que causa demora no correto diagnóstico e, consequentemente, no tratamento ${ }^{1}$. Exames de imagem, como no caso apresentado neste trabalho, são de fundamental importância, uma vez que servem de diagnóstico diferencial e auxiliam na determinação do correto diagnóstico. 


\section{Considerações finais}

A limitação de abertura bucal nem sempre é decorrente de alterações articulares, ela pode estar relacionada à hiperplasia do processo coronoide. A técnica cirúrgica da coronoidectomia por acesso intraoral é rápida, pouco traumática e eficaz no tratamento das hiperplasias coronoideas. No entanto, essa condição ainda deve ser mais estudada, a fim de que se compreendam melhor seus fatores etiológicos e, assim, se estabeleça um diagnóstico precoce, para que uma terapia adequada possa ser aplicada, permitindo ao paciente uma melhor qualidade de vida.

\section{Abstract}

The coronoid process hyperplasia is an unusual condition of unknown etiology that is presented clinically through mouth opening limitation, without painful symptoms during mouth opening and closure. Objective: to report and discuss, through a surgical case, the treatment of mouth opening limitation caused by bilateral coronoid process hyperplasia. Case report: female patient, 11 years old, referred due to chewing difficulty by mouth opening limitation. No history of trauma in the face or joint area. The tomographic examination showed the bilateral elongation of the coronoid process, causing it to collide with the zygomatic arch during mouth opening, which caused locking. The treatment proposed was bilateral coronoidectomy with intraoral surgical access, which enhanced mouth opening at the immediate postoperative period. Final considerations: coronoidectomy is a surgical approach with easy intraoral access, non-traumatic, and effective in the treatment of patients with coronoid process hyperplasia.

Keywords: Coronoidectomy. Coronoid process hyperplasia. Mouth opening limitation.

\section{Referências}

1. McLoughlin PM, Hopper C, Bowley NB. Hyperplasia of the mandibular coronoid process: an analysis of 31 cases and a review of the literature. J Oral Maxillofac Surg $1995 ; 53(3): 250-5$.

2. Isberg A, Isacsson G, Nah KS. Mandibular coronoid process locking: a prospective study of frequency and association with internal derangement of the temporomandibular joint. Oral Surg Oral Med Oral Pathol 1987; 63(3):275-9.

3. Loh HS, Ling SY, Lian CB, Shanmuhasuntharam P. Bilateral coronoid hyperplasia - a report with a view on its management. J Oral Rehabil 1997; 24(10):782-7.

4. Rowe NL. Bilateral developmental hyperplasia of the mandibular coronoid process: a report of two cases. Br J Oral Surg 1963; 1:90-104.

5. Lyon LZ, Sarnat BG. Limited opening of the mouth caused by enlarged coronoid processes: report of case. J Am Dent Assoc 1963; 67(5):644-50.

6. York BV, Cockerham S. Bilateral hyperplasia of the coronoid processes in siblings. Oral Surg 1983; 56(6):584-5.
7. Shira RB, Lister RL. Limited mandibular movements due to enlargement of the coronoid processes. J Oral Surg 1958; 16:183.

8. Neville BW, Damm DD, Allen CM, Bouquot JS. Patologia oral e maxilofacial. 2. ed. Rio de Janeiro: Guanabara Koogan; 2004.

9. Praal FR. Limitation of mandibular movement due to bilateral mandibular coronoid process enlargement. J Oral Maxillof Surg 1984; 42(8):534-6.

10. Takahashi A, Hao-Zong W, Murakami S, Kondoh H, Fujishita M, Fuchihata H. Diagnosis of coronoid process hyperplasia by three-dimensional computed tomographic imaging. Dentomaxillofac Radiol 1993; 22(3):149-54.

11. Gerbino G, Bianchi SD, Bernardi M, Berrone S. Hyperplasia of the mandibular coronoid process: long-term followup after coronoidotomy. Cranio-Maxillofacial Surg 1997; 25(3):169-73.

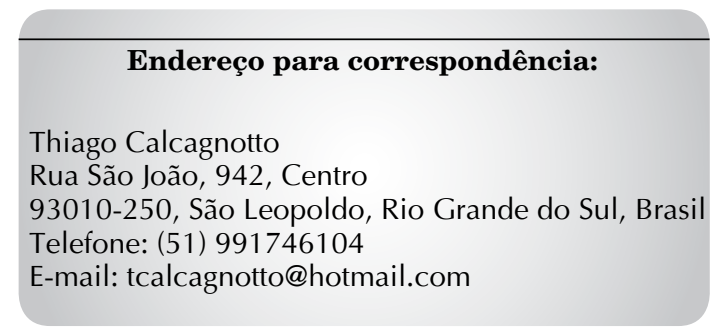

Recebido: 23/01/18. Aceito: 26/03/18. 